\title{
The Three-Dimensional Weak Form of the Conjugate Gradient FFT Method for Solving Scattering Problems
}

\author{
Peter Zwamborn and Peter M. van den Berg
}

\begin{abstract}
The problem of electromagnetic scattering by a three-dimensional dielectric object can be formulated in terms of a hypersingular integral equation, in which a grad-div operator acts on a vector potential. The vector potential is a spatial convolution of the free space Green's function and the contrast source over the domain of interest. A weak form of the integral equation for the relevant unknown quantity is obtained by testing it with appropriate testing functions. As next step the vector potential is expanded in a sequence of the appropriate expansion functions and the grad-div operator is integrated analytically over the scattering object domain only. A weak form of the singular Green's function has been used by introducing its spherical mean. As a result, the spatial convolution can be carried out numerically using a trapezoidal integration rule. This method shows excellent numerical performance.
\end{abstract}

\section{INTRODUCTION}

$\mathrm{D}$ URING the past several years considerable effort has been put into the development of computational techniques for handling the scattering and diffraction of electromagnetic waves by an object. We can distinguish between global techniques (e.g., the use of wave function expansions and integral equations) and local techniques (finite-difference and finite-elements methods). One of the extensively utilized and most versatile global methods is the domain-integral-equation technique. It takes into account that the irradiated object is present in free space and that it manifests itself through the presence of secondary sources of contrast currents. Numerous methods have been developed, and it is not our objective to survey them all. Instead we concentrate on the $k$-space methods. It is our opinion that methods of this type are applicable for threedimensional electromagnetic scattering problems owing to their storage and computational efficiency.

The problem of the electromagnetic scattering by an inhomogeneous dielectric object is formulated in terms of an integral equation for the electric field over the domain

\footnotetext{
Manuscript received September 11, 1991; revised January 29, 1992. This work was supported through a NATO Grant-0230/88 and Research Grants from the Stichting Fund for Science, Technology and Research (a companion organization to the Schlumberger Foundation in the U.S.A.), from Schlumberger-Doll Research, Ridgefield, CT, and from Etudes et Productions Schlumberger, Clamart, France.

The authors are with the Laboratory for Electromagnetic Research, Department of Electrical Engineering, Delft University of Technology, P.O. Box 5031, 2600 GA Delft. The Netherlands.

IEEE Log Number 9201713
}

of the object. The first method for solving the electricfield integral equation over the domain of a dielectric object was developed by Richmond for the two-dimensional TM case [1], and for the two-dimensional TE case [2]. Here the method of moments has been used with pulse expansion functions and point matching. The method of moments requires the inversion of a (large) matrix, limiting the application of this method. This problem has been circumvented by using a conjugate gradient iterative technique [3], [4]. Bojarski has introduced the $k$-space method, obtaining an iterative approach that reduces the storage and the computation time by using a Fast Fourier Transform algorithm for the computation of the spatial convolution that occurs in the integral equation. A comprehensive review of Bojarski's work, together with the appropriate references to his $k$-space frequency domain method, can be found in his $1982 k$-space time domain paper [5]. Subsequently, the conjugate gradient method combined with the Fast Fourier Transform has been developed for various configurations [6]-[16]. For the threedimensional problems and the two-dimensional case of TE polarization, applicability of this conjugate gradient FFT method using pulse expansion functions casts some serious doubts [17]-[19]. The operator involved consists of a grad-div operator that acts on a vector potential. The vector potential is an integral of the product of a Green's function and the electric contrast current density inside the scattering object. The vector potential is a spatial convolution. In the spectral Fourier domain this convolution is algebraic: a simple product. Recently, the weak formulation of the conjugate gradient FFT method has proved to be an efficient and accurate scheme for solving twodimensional TE scattering by strongly inhomogeneous lossy dielectric objects [20]. Therefore, in this present paper, we present a weak formulation of the domain-integral equation for the modeling of full vectorial, three-dimensional, electromagnetic scattering problems. The domainintegral equation that is obtained in its strong form is weakened by testing it with appropriate testing functions. This weak form is the operator equation to be solved by a CGFFT method. The advantages of this procedure are, firstly, that the grad-div operator acting on the vector potential is integrated analytically over the domain of the dielectric object only and, secondly, that we have main- 
tained the simple scalar form of the convolution structure of the vector potential (in fact three scalar convolutions). The integral equation is formulated in terms of the unknown electric flux density rather than in terms of the electric field strength. The continuity of the normal component of the electric flux density yields a correct implementation of the boundary condition of the normal component of the electric field at the interfaces of (strong) discontinuity. As a consequence, the present scheme is much simpler than the one of Joachimowizc and Pichot [21]. No surface integrals that are directly related to surface charges have to be introduced. Further, it should be mentioned that expanding the electric-contrast vector potential directly, as opposed to other schemes where only the current density is expanded such as the CGM-FFT scheme of Catedra et al. [16], leads to a weaker singularity in the Green's function. The latter aspect gives rise to a more accurate numerical evaluation of the (convolution type) integral operators involved. In contrast to the weak formulation of the two-dimensional TE-case [20], the three-dimensional formulation is presented for different mesh-sizes in the three Cartesian coordinates.

Finally, it is noted that the continuous convolution of the Green's function with the contrast current density is replaced by a discrete cyclic convolution that can be evaluated with a period in the FFT as small as possible. The Green's function is the point source solution of a scalar wave equation. Instead of using this strong form, we employ its spherical mean, being the normalized integrated value over some small spherical region. The radius of this spherical region is directly related to the mesh size of the discretized configuration.

We present some numerical results for three-dimensional problems. Numerical computations have been carried out for a strongly inhomogeneous, lossy radially layered sphere. These numerical results are compared with existing analytical solutions (Mie series) and it is directly observed that the weak form of the conjugate gradient FFT method yields excellent results. As second test case, the bistatic radar cross section of a conducting thin slab is compared with the bistatic radar cross section of a perfectly conducting plate. It is demonstrated that for both configurations comparable results have been obtained. Further, the numerical far-field results for some cubical configurations are compared with results recently published in the literature.

These test cases demonstrate that the present weak formulation of the conjugate gradient FFT method can be considered to be a comparitively simple and efficient tool for solving scattering problems pertaining to (strongly) inhomogeneous lossy dielectric objects.

\section{The Domain-Integral Equation}

The vectorial position in the three-dimensional space is denoted by $\boldsymbol{x}=\left(x_{1}, x_{2}, x_{3}\right)$. The unit vectors in the $x_{1^{-}}$, $x_{2^{-}}$, and $x_{3}$-directions are given by $i_{1}, i_{2}$ and $i_{3}$. The time factor $\exp (-i \omega t)$ has been used for the field quantities in the frequency-domain. We consider the problem of scattering by a lossy inhomogeneous dielectric object with complex permittivity

$$
\epsilon(\boldsymbol{x})=\epsilon_{r}(\boldsymbol{x}) \epsilon_{0}+i \frac{\sigma(\boldsymbol{x})}{\omega},
$$

where $\epsilon_{r}$ denotes the relative permittivity of the object with respect to the lossless and homogeneous embedding with permittivity $\epsilon_{0}$, and where $\sigma$ denotes the electric conductivity of the object. The incident electric field is denoted as $\boldsymbol{E}^{i}=\left(E_{1}^{i}, E_{2}^{i}, E_{3}^{i}\right)$. In this paper, we formulate the scattering problem as a domain-integral equation for the unknown electric flux density $\boldsymbol{D}=\left(D_{1}, D_{2}, D_{3}\right)$ over the object domain $E^{S}$ as

$$
\left.\boldsymbol{E}^{i}(\boldsymbol{x})=\frac{\boldsymbol{D}(\boldsymbol{x})}{\epsilon(\boldsymbol{x})}-\left(k_{0}^{2}+\operatorname{grad} \operatorname{div}\right) A(\boldsymbol{x}), \quad \boldsymbol{x} \in \mathbb{D}\right)^{S} .
$$

where $k_{0}=\omega\left(\epsilon_{0} \mu_{0}\right)^{1 / 2}$ and the vector potential $\boldsymbol{A}=\left(A_{1}\right.$, $\left.A_{2}, A_{3}\right)$ is given by

$$
\boldsymbol{A}(\boldsymbol{x})=\frac{1}{\epsilon_{0}} \int_{x^{\prime} \in \mathrm{s}} G\left(\boldsymbol{x}-\boldsymbol{x}^{\prime}\right) \chi\left(x^{\prime}\right) \boldsymbol{D}\left(\boldsymbol{x}^{\prime}\right) d \boldsymbol{x}^{\prime}
$$

in which the normalized contrast function $\chi$ is defined as

$$
\chi(x)=\frac{\epsilon(x)-\epsilon_{0}}{\epsilon(x)} .
$$

Further, the three-dimensional Green's function $G$ is given by

$$
G(x)=\frac{\exp \left(i k_{0}|x|\right)}{4 \pi|x|}, \quad x \in \mathbb{R}^{3} .
$$

\section{Testing and Expansion Procedure}

We first introduce a discretization in the spatial domain $\boldsymbol{x}=\left(x_{1}, x_{2}, x_{3}\right)$. We use a uniform mesh with grid widths of $\Delta x_{1}, \Delta x_{2}$ and $\Delta x_{3}$ in the $x_{1}, x_{2}$ and $x_{3}$ directions, respectively. For our convenience the discrete values of $x$ are given by

$$
\boldsymbol{x}_{M, N, P}=\left\{\left(M-\frac{1}{2}\right) \Delta x_{1},\left(N-\frac{1}{2}\right) \Delta x_{2},\left(P-\frac{1}{2}\right) \Delta x_{3}\right\},
$$

denoting the centerpoints of the volumetric subdomains. The upper-case Latin subscript are bounded, viz. $M \in[1$, $\left.B_{1}\right], N \in\left[1, B_{2}\right]$ and $P \in\left[1, B_{3}\right]$. The scatterer domain is completely embedded in the rectangular block with dimension $B_{1} \Delta x_{1} \times B_{2} \Delta x_{2} \times B_{3} \Delta x_{3}$. The boundary of the discretized object now consists of surfaces parallel to the $x_{1-}, x_{2^{-}}$, or $x_{3}$-axis. We assume that the discretized boundary $\partial{ }^{S}$ of the scattering domain ${ }^{S}$ lies completely in the embedding where $\chi=0$. This is always possible, since we can extend the definition of the scattering domain $\partial^{S}$ by extending it with a zero contrast function $\chi$. In each volumetric subdomain with center $\boldsymbol{x}_{M, N, P}$ and dimension $\Delta x_{1} \times \Delta x_{2} \times \Delta x_{3}$, we assume the complex permittivity to be constant with values $\epsilon_{M, N, P}$. Note that 
jumps in the (complex) permittivity function may occur at $x_{1}=M \Delta x_{1}, x_{2}=N \Delta x_{2}$ and $x_{3}=P \Delta x_{3}$.

In order to cope with the grad-div operator in (2), we test the strong form of (2) by multiplying both sides of the equality sign by a vectorial testing function $\boldsymbol{\psi}_{M, N, P}^{(p)}(\boldsymbol{x}), p=1,2,3$, and integrate the result over the domain $x \in \mathbb{D}^{S}$. The testing function $\boldsymbol{\psi}_{M, N, P}^{(p)}(\boldsymbol{x})=$ $\psi_{M, N, P}^{(p)}(x) i_{p}$ is a suitably chosen vectorial function that will be defined later. We then obtain

$$
\begin{aligned}
\int_{x \in \mathbb{Q} S} & \psi_{M, N, P}^{(p)}(x) E_{p}^{i}(x) d x \\
= & \int_{x \in \unrhd S} \psi_{M, N, P}^{(p)}(x) \frac{D_{p}(x)}{\epsilon(x)} d x \\
& -k_{0}^{2} \int_{x \in \mathbb{Q}} \psi_{M, N, P}^{(p)}(\boldsymbol{x}) A_{p}(x) d x \\
& +\int_{x \in \mathbb{Q}} \partial_{p} \psi_{M, N, P}^{(p)}(\boldsymbol{x}) \operatorname{div} \boldsymbol{A}(\boldsymbol{x}) d \boldsymbol{x}
\end{aligned}
$$

for $p=1,2,3$, and where we have used Gauss' theorem on each subdomain where $\partial_{p} \psi_{M, N, P}^{(p)}(\boldsymbol{x}) \operatorname{div} \boldsymbol{A}(\boldsymbol{x})$ is continuously differentiable and by using the continuity of the normal components of this function through the interfaces between these subdomains. In view of the derivation of (7), it is mentioned that for the testing functions $\psi_{M, N, P}^{(p)}(\boldsymbol{x})$, the partial derivative $\partial_{p} \psi_{M, N, P}^{(p)}(\boldsymbol{x})$ must be piecewise continuous on the domain $S$. At the surfaces (with normal $v$ ) where this property fails, we then require that $\boldsymbol{v} \cdot \boldsymbol{\psi}_{M, N, P}^{(p)}(\boldsymbol{x})$ must be continuous. Further, $\boldsymbol{v} \cdot$ $\boldsymbol{\psi}_{M, N, P}^{(p)}(\boldsymbol{x})$ must vanish for $\boldsymbol{x} \notin \mathrm{S}^{S}$. We expand the generalized electric flux density, the electric-contrast vector potential and the incident electric field in a sequence of vectorial expansion functions $\psi_{I, J, K}^{(q)}(x)=\psi_{I, J, K}^{(q)}(\boldsymbol{x}) i_{q}, q=$ $1,2,3$ as follows

$$
\begin{array}{ll}
D_{q}(x)=\epsilon_{0} \sum_{I, J, K} d_{l, J, K}^{(q)} \psi_{I, J, K}^{(q)}(x) & \text { for } x \in S^{S}, \\
A_{q}(x)=\sum_{I, J, K} A_{I, J, K}^{(q)} \psi_{I, J, K}^{(q)}(x) & \text { for } x \in \mathbb{D}^{S}, \\
E_{q}^{i}(x)=\sum_{I, J, K} E_{I, J, K}^{i,(q)} \psi_{I, J, K}^{(q)}(x) & \text { for } x \in D^{S}
\end{array}
$$

Substitution of (8)-(10) in (7), carrying out the divergence and interchanging the order of summation and integration, we obtain the following weak form of the domain-integral equation

$$
\begin{aligned}
e_{M, N, P}^{i,(p)}= & \sum_{I, J, K} \sum_{q=1}^{3} \boldsymbol{d}_{I, J, K}^{(q)} \boldsymbol{u}_{M, N, P ; I, J, K}^{(p, q)} \\
& +\boldsymbol{A}_{I, J, K}^{(q)}\left[-\boldsymbol{k}_{0}^{2} \boldsymbol{v}_{M, N, P, I, J, K}^{(p, q)}+\boldsymbol{w}_{M, N, P ; I, J, K}^{(p, q)}\right]
\end{aligned}
$$

in which

$$
e_{M, N, P}^{i,(p)}=\sum_{I, J, K} \sum_{q=1}^{3} E_{I, J, K}^{i,(q)} v_{M, N, P ; I, J, K}^{(p, q)}
$$

$u_{M, N, P ; I, J, K}^{(p, q)}=\delta_{p, q} \int_{x \in \mathbb{D} S} \psi_{M, N, P}^{(p)}(x) \frac{\epsilon_{0}}{\epsilon(\boldsymbol{x})} \psi_{I, J, K}^{(q)}(\boldsymbol{x}) d \boldsymbol{x}$

$v_{M, N, P ; I, J, K}^{(p, q)}=\delta_{p, q} \int_{x \in \mathbb{Q} S} \psi_{M, N . P}^{(p)}(x) \psi_{I, J, K}^{(q)}(\boldsymbol{x}) d \boldsymbol{x}$,

$w_{M, N, P ; I, J, K}^{(p, q)}=\int_{x \in \mathbb{D} S} \partial_{p} \psi_{M, N, P}^{(p)}(\boldsymbol{x}) \partial_{q} \psi_{I, J, K}^{(q)}(x) d \boldsymbol{x}$,

for $p=1,2,3$ and where $\delta_{p, q}$ denotes the Kronecker symbol.

In view of the partial derivatives in (15), the volumetric rooftop functions [23] are chosen as testing and expansion functions, viz.

$$
\begin{aligned}
\psi_{M, N, P}^{(1)}(\boldsymbol{x})= & \Lambda\left(x_{1}-x_{1, M, N, P}+\frac{1}{2} \Delta x_{1} ; 2 \Delta x_{1}\right) \\
& \cdot \Pi\left(x_{2}-x_{2 ; M, N, P} ; \Delta x_{2}\right) \\
& \times \Pi\left(x_{3}-x_{3: M, N, P} ; \Delta x_{3}\right), \\
\psi_{M, N, P}^{(2)}(\boldsymbol{x})= & \Pi\left(x_{1}-x_{1 ; M, N, P} ; \Delta x_{1}\right) \\
& \cdot \Lambda\left(x_{2}-x_{2: M, N, P}+\frac{1}{2} \Delta x_{2} ; 2 \Delta x_{2}\right) \\
& \times \Pi\left(x_{3}-x_{3: M, N, P} ; \Delta x_{3}\right), \\
\psi_{M, N, P}^{(3)}(\boldsymbol{x})= & \Pi\left(x_{1}-x_{1 ; M, N, P} ; \Delta x_{1}\right) \\
& \cdot \Pi\left(x_{2}-x_{2 ; M, N, P} ; \Delta x_{2}\right) \\
& \times \Lambda\left(x_{3}-x_{3: M, N, P}+\frac{1}{2} \Delta x_{3} ; 2 \Delta x_{3}\right),
\end{aligned}
$$

in which $\Lambda=\Lambda(y ; 2 \Delta x)$ is the one-dimensional piecewise linear and continuous function, viz. the triangle function with support $2 \Delta x$, and $\Pi=\Pi(y ; \Delta x)$ is the one-dimensional piecewise constant function, viz. the pulse function with support $\Delta x$.

Using these functions of (16)-(18) in (12)-(15), we obtain the following weak formulation of the domain-integral equation:

$$
\begin{aligned}
e_{M, N, P}^{i,(1)}= & \sum_{I=1}^{3}\left[\boldsymbol{b}_{I}^{(1)} \boldsymbol{d}_{M+I-2, N, P}^{(1)}+\boldsymbol{c}_{I}^{(1)} \boldsymbol{A}_{M+I-2, N . P}^{(1)}\right] \\
& +\sum_{I=1}^{2} \sum_{J=1}^{2} t_{I, J}^{(3)} \boldsymbol{A}_{M+I-2, N+J-1, P}^{(2)} \\
& +\sum_{I=1}^{2} \sum_{K=1}^{2} \boldsymbol{t}_{I, K}^{(2)} \boldsymbol{A}_{M+I-2, N, P+K-1}^{(3)}, \\
e_{M, N, P}^{i,(2)}= & \sum_{I=1}^{2} \sum_{J=1}^{2} \boldsymbol{t}_{J, I}^{(3)} \boldsymbol{A}_{M+I-1, N+J-2, P}^{(1)} \\
& +\sum_{J=1}^{3}\left[\boldsymbol{b}_{J}^{(2)} \boldsymbol{d}_{M, N+J-2, P}^{(2)}+\boldsymbol{c}_{J}^{(2)} \boldsymbol{A}_{M, N+J-2, P}^{(2)}\right] \\
& +\sum_{J=1}^{2} \sum_{K=1}^{2} \boldsymbol{t}_{J, K}^{(1)} \boldsymbol{A}_{M, N+J-2, P+K-1}^{(3)}
\end{aligned}
$$




$$
\begin{aligned}
\boldsymbol{e}_{M, N, P}^{i_{M}(3)}= & \sum_{I=1}^{2} \sum_{K=1}^{2} \boldsymbol{t}_{K, I}^{(2)} \boldsymbol{A}_{M+1-1, N, P+K-2}^{(1)} \\
& +\sum_{J=1}^{2} \sum_{K=1}^{2} \boldsymbol{t}_{K, J}^{(1)} \boldsymbol{A}_{M, N+J-1, P+K-2}^{(2)} \\
& +\sum_{K=1}^{3}\left[\boldsymbol{b}_{K}^{(3)} \boldsymbol{d}_{M, N, P+K-2}^{(3)}+c_{K}^{(3)} \boldsymbol{A}_{M, N, P+K-2}^{(3)}\right],
\end{aligned}
$$

in which the coefficients of the vectors $\boldsymbol{b}^{(p)}$ and $\boldsymbol{c}^{(p)}$ are obtained as

$$
\begin{aligned}
& \boldsymbol{b}^{(1)}=\frac{\Delta x_{1} \Delta x_{2} \Delta x_{3}}{6}\left(\begin{array}{l}
\frac{\epsilon_{0}}{\epsilon_{M-1, N, P}} \\
\frac{2 \epsilon_{0}}{\epsilon_{M-1, N, P}}+\frac{2 \epsilon_{0}}{\epsilon_{M, N, P}} \\
\frac{\epsilon_{0}}{\epsilon_{M, N, P}}
\end{array}\right), \\
& \boldsymbol{b}^{(2)}=\frac{\Delta x_{1} \Delta x_{2} \Delta x_{3}}{6}\left(\begin{array}{l}
\frac{\epsilon_{0}}{\epsilon_{M, N-1, P}} \\
\frac{2 \epsilon_{0}}{\epsilon_{M, N-1, P}}+\frac{2 \epsilon_{0}}{\epsilon_{M, N, P}} \\
\frac{\epsilon_{0}}{\epsilon_{M, N . P}}
\end{array}\right) \text {, } \\
& \boldsymbol{b}^{(3)}=\frac{\Delta x_{1} \Delta x_{2} \Delta x_{3}}{6}\left(\begin{array}{l}
\frac{\epsilon_{0}}{\epsilon_{M, N, P-1}} \\
\frac{2 \epsilon_{0}}{\epsilon_{M, N, P-1}}+\frac{2 \epsilon_{0}}{\epsilon_{M, N, P}} \\
\frac{\epsilon_{0}}{\epsilon_{M, N, P}}
\end{array}\right), \\
& c^{(p)}=\Delta x_{1} \Delta x_{2} \Delta x_{3} \\
& {\left[-\frac{k_{0}^{2}}{6}\left(\begin{array}{l}
1 \\
4 \\
1
\end{array}\right)+\left(\Delta x_{p}\right)^{-2}\left(\begin{array}{r}
-1 \\
2 \\
-1
\end{array}\right)\right],}
\end{aligned}
$$

while the coefficients of the matrix $t^{(p)}$ follow from

$$
\boldsymbol{t}^{(p)}=\Delta x_{p}\left(\begin{array}{rr}
-1 & 1 \\
1 & -1
\end{array}\right) .
$$

The values of $e_{M, N, P}^{i,(p)}$ follow from $E_{M, N, P}^{i,(p)}$ as

$$
\begin{aligned}
e_{M, N, P}^{i,(1)}= & \frac{\Delta x_{1} \Delta x_{2} \Delta x_{3}}{6} \\
& \cdot\left[E_{M+1, N, P}^{i,(1)}+4 E_{M, N, P}^{i,(1)}+E_{M-1, N, P}^{i,(1)}\right],
\end{aligned}
$$

$$
\begin{aligned}
e_{M, N, P}^{i,(2)}= & \frac{\Delta x_{1} \Delta x_{2} \Delta x_{3}}{6} \\
& \cdot\left[E_{M, N+1, P}^{i,(2)}+4 E_{M, N, P}^{i,(2)}+E_{M, N-1, P}^{i,(2)}\right], \\
e_{M, N, P}^{i,(3)}= & \frac{\Delta x_{1} \Delta x_{2} \Delta x_{3}}{6} \\
& \cdot\left[E_{M, N, P+1}^{i,(3)}+4 E_{M, N, P}^{i,(3)}+E_{M, N, P-1}^{i,(3)}\right] .
\end{aligned}
$$

With our particular choice of expansion functions, the quantities $A_{M . N, P}^{(p)}$ and $e_{M, N, P}^{i,(p)}$ follow from

$$
\begin{gathered}
d_{M, N, P}^{(p)}=\frac{D_{p}\left(x_{M, N, P}-\frac{1}{2} \Delta x_{p} i_{p}\right)}{\epsilon_{0}}, \\
p=1,2,3, \\
A_{M, N, P}^{(p)}=A_{p}\left(x_{M, N . P}-\frac{1}{2} \Delta x_{p} i_{p}\right), \\
p=1,2,3, \\
E_{M, N, P}^{i,(p)}=E_{p}^{i}\left(x_{M, N, P}-\frac{1}{2} \Delta x_{p} i_{p}\right), \\
p=1,2,3 .
\end{gathered}
$$

The electric-contrast vector potential $A_{m}$ is related to the electric flux density $D$ via (3). Note that with this procedure we have enforced the equality sign of (30)-(32) exactly in a single point. Again, this is a strong form and we will weaken this form by taking the spherical mean. The computation of the electric contrast vector potential is discussed in next paragraph.

Let us define the spherical mean (weak form) of the electric-constant vector potential as

$$
[A](x)=\frac{\int_{\left|x^{\prime \prime}\right|<(1 / 2) \Delta x} A\left(x+x^{\prime \prime}\right) d x^{\prime \prime}}{\int_{\left|x^{\prime \prime}\right|<(1 / 2) \Delta x} d x^{\prime \prime}},
$$

where $\Delta x=\min \left[\Delta x_{1}, \Delta x_{2}, \Delta x_{3}\right]$. Substitution of (3) in (33) and interchanging the order of integrations, we obtain

$$
[\boldsymbol{A}](\boldsymbol{x})=\frac{1}{\epsilon_{0}} \int_{x^{\prime} \in Q}[G]\left(\boldsymbol{x}-\boldsymbol{x}^{\prime}\right) \chi\left(\boldsymbol{x}^{\prime}\right) D\left(\boldsymbol{x}^{\prime}\right) d \boldsymbol{x}^{\prime},
$$

in which

$$
[G](x)=\frac{\int_{\left|x^{\prime \prime}\right|<(1 / 2) \Delta x} G\left(x+x^{\prime \prime}\right) d x^{\prime \prime}}{\int_{\left|x^{\prime \prime}\right|<(1 / 2) \Delta x} d x^{\prime \prime}} .
$$

Taking the spherical mean of the electric-contrast vector potential, the integral of (35) can be determined analytically. Using spherical coordinates together with the addition theorem of the modified spherical Bessel functions, 
it is easily verified that

$$
[G](x)= \begin{cases}\frac{\left(1-\frac{1}{2} i k_{0} \Delta x\right) \exp \left(\frac{1}{2} i k_{0} \Delta x\right)-1}{\frac{1}{6} \pi k_{0}^{2}(\Delta x)^{3}} & \text { if }|\boldsymbol{x}|=0, \\ \frac{\exp \left(i k_{0}|x|\right)\left[\frac{\sinh \left(\frac{1}{2} i k_{0} \Delta x\right)}{\frac{1}{2} i k_{0} \Delta x}-\cosh \left(\frac{1}{2} i k_{0} \Delta x\right)\right]}{\frac{1}{3} \pi\left(k_{0} \Delta x\right)^{2}|x|} & \text { if }|\boldsymbol{x}|>\frac{1}{2} \Delta x .\end{cases}
$$

Note that, for the limiting case $\Delta x \rightarrow 0$, the weak form of the Green's function $[G](x),|x|>\frac{1}{2} \Delta x$, tends to the strong form of the Green's function $G(x)$.

As next step, the continuous convolution integral of (34) has to be replaced by a discrete one. Using a trapezoidal integration rule, we arrive at

$$
\begin{aligned}
A_{M . N, P}^{(p)}= & \Delta x_{1} \Delta x_{2} \Delta x_{3} \sum_{M^{\prime}, N^{\prime}, P^{\prime}} \\
& \cdot G_{M-M^{\prime}, N-N^{\prime}, P-P^{\prime}} \chi_{M^{\prime}, N^{\prime}, P^{\prime}}^{(p)} d_{M^{\prime}, N^{\prime}, P^{\prime}}^{(p)},
\end{aligned}
$$

in which $p=1,2,3$; the discrete values of the normalized contrast function follow from

$$
\begin{aligned}
& \chi_{M, N, P}^{(1)}=\frac{\chi\left(x_{M-1, N, P}\right)+\chi\left(x_{M, N, P}\right)}{2}, \\
& \chi_{M, N, P}^{(2)}=\frac{\chi\left(x_{M, N-1, P}\right)+\chi\left(x_{M, N, P}\right)}{2}, \\
& \chi_{M, N, P}^{(3)}=\frac{\chi\left(x_{M, N, P-1}\right)+\chi\left(x_{M, N, P}\right)}{2} .
\end{aligned}
$$

The discrete values of the weakened Green's function are given by

$$
G_{M, N, P}=[G]\left(M \Delta x_{1}, N \Delta x_{2}, P \Delta x_{3}\right) .
$$

Using the convolution theorem of discrete Fourier transform (DFT), (37) is evaluated numerically by

$$
\begin{aligned}
A_{M, N, P}^{(p)}= & \Delta x_{1} \Delta x_{2} \Delta x_{3} \mathrm{DFT}^{-1}\left\{\mathrm{DFT}\left\{[G]_{M^{\prime}, N^{\prime}, P^{\prime}}\right\}\right. \\
& \left.\cdot \operatorname{DFT}\left\{\chi_{M, N, P}^{(p)} d_{M, N, P}^{(p)}\right\}\right\} .
\end{aligned}
$$

Note that the subscripts $M^{\prime}, N^{\prime}$, and $P^{\prime}$ of $[G]_{M^{\prime} . N^{\prime} . P^{\prime}}$ in (42) are dictated by (6) and the spatial periodicity of the discrete Fourier transform.

Let us assume that the domain of the object lies completely inside a block

$$
\begin{aligned}
& \left(M_{\min }-1\right) \Delta x_{1}<x_{1}<\left(M_{\max }+1\right) \Delta x_{1}, \\
& \left(N_{\min }-1\right) \Delta x_{2}<x_{2}<\left(N_{\max }+1\right) \Delta x_{2}, \\
& \left(P_{\min }-1\right) \Delta x_{3}<x_{3}<\left(P_{\max }+1\right) \Delta x_{3} .
\end{aligned}
$$

In this volumetric domain we have $M_{D^{\mathrm{s}}}=M_{\max }-M_{\min }$ +1 meshpoints in the $x_{1}$-direction, $N_{D^{\mathrm{s}}}=N_{\max }-N_{\min }$ +1 meshpoints in the $x_{2}$-direction and $P_{D^{\mathrm{s}}}=P_{\max }-P_{\min }$ +1 meshpoints in the $x_{3}$-direction. Based on the ideas of Barkeshili and Volakis [24], it is easily shown [25] that (37) is equivalent to (42) inside the object domain $S$ if the relevant DFT's are defined inside a block with $M_{\mathrm{DFT}}$ meshpoints in the $x_{1}$-direction, $N_{\mathrm{DFT}}$ meshpoints in the $x_{2}$-direction and $P_{\mathrm{DFT}}$ meshpoints in the $x_{3}$-direction, such that

$$
\begin{aligned}
M_{D F T} & \geq 2\left(M_{D^{\mathrm{s}}}+1\right), \quad N_{D F T} \geq 2\left(N_{D^{\mathrm{s}}}+1\right), \\
P_{D F T} & \geq 2\left(P_{D^{\mathrm{s}}}+1\right) .
\end{aligned}
$$

Finally, the quantity $E_{M, N, P}^{i,(p)}$ is given in case the incident field is taken to be a uniform plane wave. Then, $\boldsymbol{E}^{i}$ follows from

$$
\boldsymbol{E}^{i}(\boldsymbol{x})=\boldsymbol{\varepsilon} \exp \left(i k_{0} \boldsymbol{\theta} \cdot \boldsymbol{x}\right),
$$

in which $\varepsilon$ denotes the amplitude of the plane wave and $\theta$ denotes the unit vector of the direction of propagation. The spherical mean (weak form) of the uniform plane wave incident field is now defined as

$$
\left[\boldsymbol{E}^{i}\right](\boldsymbol{x})=\frac{\int_{\left|\boldsymbol{x}^{\prime \prime}\right|<(1 / 2) \Delta x} \boldsymbol{E}^{i}\left(\boldsymbol{x}+\boldsymbol{x}^{\prime \prime}\right) d \boldsymbol{x}^{\prime \prime}}{\int_{\left|x^{\prime \prime}\right|<(1 / 2) \Delta x} d \boldsymbol{x}^{\prime \prime}}
$$

The weak form of the incident uniform plane wave is obtained by substitution of (47) in (48) as follows

$$
\begin{aligned}
{\left[\boldsymbol{E}^{i}\right](\boldsymbol{x})=} & \varepsilon \exp \left(i k_{0} \boldsymbol{\theta} \cdot \boldsymbol{x}\right) \frac{12}{\left(k_{0} \Delta x\right)^{2}} \\
& {\left[\frac{\sinh \left(\frac{1}{2} i k_{0} \Delta x\right)}{\frac{1}{2} i k_{0} \Delta x}-\cosh \left(\frac{1}{2} i k_{0} \Delta x\right)\right] }
\end{aligned}
$$

The latter weak form gives the representations for the quantity $E_{M, N, P}^{i,(p)}$ as

$$
E_{M, N, P}^{i,(p)}=\left[E_{p}^{i}\right]\left(x_{M, N . P}-\frac{1}{2} \Delta x_{p} i_{p}\right) .
$$

Note that, for the limiting case $\Delta x \rightarrow 0$ the strong form of the incident uniform plane wave is obtained (cf. (47)).

Collecting all the results, the weak form of the domainintegral equation is given by (19)-(29), (42) and (50). This domain integral equation is symbolically written as

$$
e^{i}=L d \text {. }
$$

The latter operator equation is solved numerically by applying a conjugate gradient iterative scheme, where the DFT's are computed efficiently using fast Fourier transform (FFT) algorithms. 
TABLE I

Computation Time and Storage of VAX 3100/76 Workstation

\begin{tabular}{ccccc}
\hline $\boldsymbol{M}_{\boldsymbol{D}^{s}} \times N_{\boldsymbol{D}^{s}} \times \boldsymbol{P}_{\boldsymbol{D}^{s}}$ & $\boldsymbol{M}_{\mathrm{DFT}} \times \boldsymbol{N}_{\mathrm{DFT}} \times \boldsymbol{P}_{\mathrm{DFT}}$ & $\begin{array}{c}\text { Number of } \\
\text { Unknowns }\end{array}$ & $\begin{array}{c}\text { CPU-time } \\
\text { of One Iteration }\end{array}$ & $\begin{array}{c}\text { Computer } \\
\text { Storage }\end{array}$ \\
\hline $7 \times 7 \times 7$ & $16 \times 16 \times 16$ & 1176 & $8 \mathrm{sec}$ & $3 \mathrm{Mb}$ \\
$15 \times 15 \times 15$ & $32 \times 32 \times 32$ & 10800 & $0.55 \mathrm{~min}$ & $5 \mathrm{Mb}$ \\
$31 \times 31 \times 31$ & $64 \times 64 \times 64$ & 92256 & $5 \mathrm{~min}$ & $18 \mathrm{Mb}$ \\
\hline
\end{tabular}

\section{Numerical Results}

The numerical convergence is measured by the normalized root-mean-square error Err:

$$
\operatorname{Err}=\frac{\left\|\boldsymbol{r}^{(n)}\right\|}{\left\|\boldsymbol{r}^{(0)}\right\|},
$$

in which $\left\|\boldsymbol{r}^{(n)}\right\|$ denotes the norm of the residual error in the satisfaction of the operator equation of (51) over the domain ${ }^{S}$ of the dielectric object in the $n$th iteration. All computations were carried out on a VAX $3100 / 76$ workstation in double precision arithmetics. The DFT's are efficiently computed using fast Fourier transform (FFT) algorithms in single precision only. The iteration process is stopped when the normalized root-mean-square error falls below $10^{-3}$. For the two-dimensional TE scattering problems, Zwamborn and van den Berg [20] have demonstrated that this strong error criterion has to be imposed. Unless explicitly specified, the incident field is taken to be a uniform plane wave with (cf. (49))

$$
\begin{aligned}
& \epsilon_{1}=1 \mathrm{~V} / \mathrm{m}, \quad \epsilon_{2}=0, \quad \epsilon_{3}=0, \\
& \theta_{1}=0, \quad \theta_{2}=0, \quad \theta_{3}=-1 .
\end{aligned}
$$

In all cases we have taken a zero initial estimate. The bistatic radar cross section follows from [26]

$$
\begin{aligned}
\operatorname{BiRCS}(\phi, \theta)= & 10 \log \left(\sigma_{\infty}(\phi, \theta)\right. \\
& -10 \log \left(\lambda_{0}^{2}\right) \mathrm{dB},
\end{aligned}
$$

in which

$$
\sigma_{\infty}(\phi, \theta)=\lim _{R \rightarrow \infty} 4 \pi R^{2} \frac{\left\|\boldsymbol{E}^{s}(\phi, \theta)\right\|^{2}}{\left\|\boldsymbol{E}^{i}(\phi, \theta)\right\|^{2}}
$$

and where $\boldsymbol{E}^{s}(\phi, \theta)$ and $\boldsymbol{E}^{i}(\phi, \theta)$ denote the scattered farfield and incident far-field vectors, respectively. In Table I, the computation time needed to evaluate one iteration on the VAX 3100/76 workstation and the number of unknowns in the scattering problem have been presented. It is noted that the VAX Fortran computer code pertaining to these values is, however, not optimized. Examining this table reveals that the computation time of each iteration is proportional to $\left(M_{\mathrm{DFT}} \times N_{\mathrm{DFT}} \times P_{\mathrm{DFT}}\right)\left[1+\log _{2}\right.$ $\left.\left(M_{\mathrm{DFT}} \times N_{\mathrm{DFT}} \times P_{\mathrm{DFT}}\right)\right]$

We firstly consider a radially layered lossy dielectric spherical object to be present with its origin at $x=\{a, a$, $a\}$, where $a$ denotes the outer radius of the sphere. It is noted that for this special test case, analytical results are obtained with the Mie series [27]. The relative permittivities and conductivities are $\epsilon_{r: 1}=72, \sigma_{1}=0.9 \mathrm{~S} / \mathrm{m}$, and

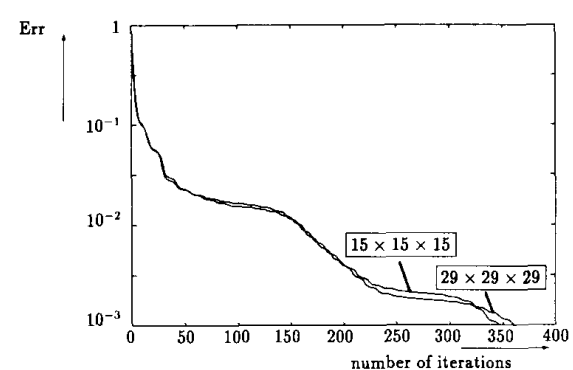

Fig. 1. The numerical convergence ratio rate obtained for the scattering by a inhomogeneous sphere; $\epsilon_{r \cdot 1}=72, \sigma_{1}=0.9 \mathrm{~S} / \mathrm{m}$ and $k_{0} a_{1}=0.163$, $\epsilon_{r: 2}=7.5, \sigma_{2}=0.05 \mathrm{~S} / \mathrm{m}$ and $k_{0} a_{2}=0.314$.

$\epsilon_{r: 2}=7.5, \sigma_{2}=0.05 \mathrm{~S} / \mathrm{m}$, respectively. The dimensions are given by $k_{0} a_{1}=0.163$ and $k_{0} a_{2}=0.314$. It is noted that $a_{1}$ denotes the radius of the inner sphere and $a_{2}$ denotes the radius of the outer sphere. The frequency of operation is taken to be $100 \mathrm{MHz}$. Note that Joachimowicz and Pichot have discussed the two-dimensional counterpart of this configuration in [21]. The computations are performed for different mesh sizes of $M_{D^{s}}=N_{D^{s}}=P_{D^{s}}$ $=15\left(M_{\mathrm{DFT}}=N_{\mathrm{DFT}}=P_{\mathrm{DFT}}=32\right)$ and $M_{D^{s}}=D^{s}=$ $P_{D^{s}}=29\left(M_{\mathrm{DFT}}=N_{\mathrm{DFT}}=P_{\mathrm{DFT}}=64\right)$, respectively. The numerical convergence rate of the iterative scheme is presented in Fig. 1, while the magnitudes of the components of the total electric field are presented in Fig. 2.

In order to investigate the discrepancies of the numerical results and the analytical results, we have taken the discretized sphere of the case $M_{D^{s}}=N_{D^{s}}=P_{D^{s}}=15$ as new object. As next step, this new object has been subdivided with $M_{D^{s}}=N_{D^{s}}=P_{D^{s}}=30$. The number of iterations to obtain an error less than 0.1 percent is 325 . From Fig. 3 it is observed that refining the mesh in the interior of the object hardly yields any improvement. The same discrepancies between the numerical results and the analytical results are observed. The latter reveals that the differences between the analytical and numerical results are caused by the block approximation of the spherical boundary. In order to obtain a better approximation of the spherical boundaries, the discretization of the sphere has to be improved.

As second test case we consider a thin slab to be present with its origin at $\boldsymbol{x}=\{a, a, b\}$, where the side length of the slab is equal to $2 a=2 \lambda_{0}\left(k_{0} a=2 \pi\right)$ and the thickness of the slab is $2 b$. Note that $\lambda_{0}$ denotes the wavelength in free space. The frequency of operation is taken to be 100 MHz. The slab is subdivided with $M_{D^{s}}=N_{D^{s}}=31$ and $P_{D^{s}}=1$ and mesh sizes $\Delta x_{1}=\Delta x_{2}=\Delta x_{3}=\left(2 \lambda_{0} / 31\right)$. 
$\left|E_{1}\right|$

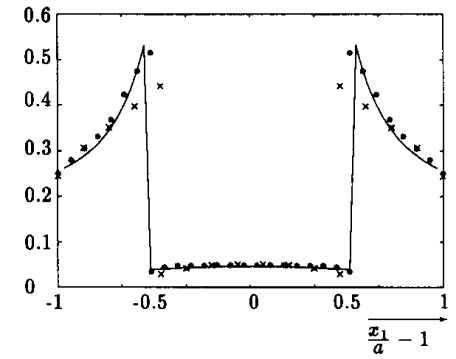

$x_{2}=a$
$x_{3}=a$

$x_{3}=a$

$\left|E_{1}\right|$

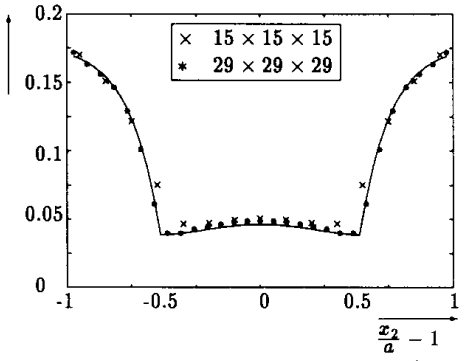

$\left|E_{3}\right|$

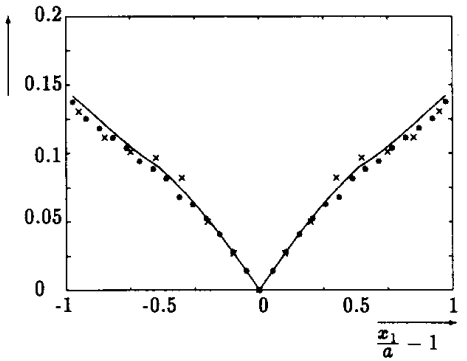

$x_{1}=a$

$x_{3}=a$
$\left|E_{1}\right|$

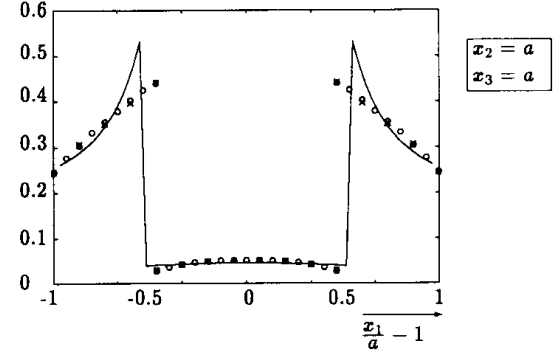

$x_{3}=a$

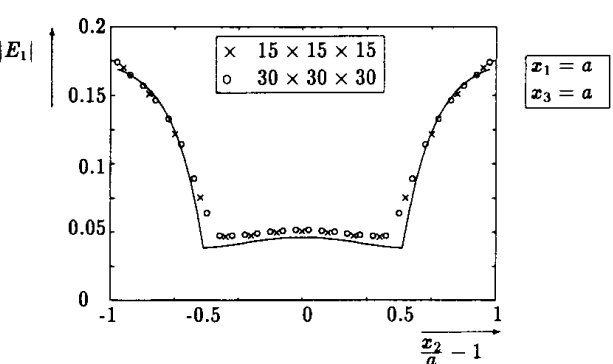

Fig. 2. The magnitude of the components of the total electric field inside a lossy inhomogeneous sphere; $\epsilon_{r: 1}=72, \sigma_{1}=0.9 \mathrm{~S} / \mathrm{m}$ and $k_{0} a_{1}=0.163$, $\epsilon_{r: 2}=7.5, \sigma_{2}=0.05 \mathrm{~S} / \mathrm{m}$ and $k_{0} a_{2}=0.314$. The numerical results pertaining to a mesh size of $15 \times 15 \times 15$ are presented by the symbols $\times$ and the numerical results pertaining to a mesh size of $31 \times 31 \times 31$ are presented by the symbols *. The analytical solution of the inhomogeneous sphere is presented by the solid line.

It is noted that the height of the slab is $2 b=\left(2 \lambda_{0} / 31\right)$. The conductivity is taken to be $1000 \mathrm{~S} / \mathrm{m}$. It is expected that the scattering from this latter object is very similar to the scattering by a perfectly conducting, infinitly thin plate. Therefore we will compare the bistatic radar cross section obtained for the slab with the bistatic radar cross section obtained for the perfectly conducting plate using the computer code of Zwamborn and van den Berg [14]. The number of iterations to obtain an error less than 0.1 percent amounts to 79 iterations for the plate configuration and 91 iterations for the slab configuration. In Fig. 4 we present the bistatic radar cross section for the perfectly conducting plate and the lossy slab in the plane $\theta=90$ $(90 \leq \phi \leq 270)$. It is observed that comparable results have been obtained for both configurations, as expected.

As third test case we consider a lossless dielectric cube to be present with its origin at $\boldsymbol{x}=\{a, a, a\}$, where the side length of the cube is equal to $2 a=0.2 \lambda_{0}\left(k_{0} a=\right.$ $0.2 \pi)$. The relative permittivity is taken to be $\epsilon_{r}=9$. The frequency of operation is taken to be $100 \mathrm{MHz}$. In this test case only, the incident field is taken to be a uniform

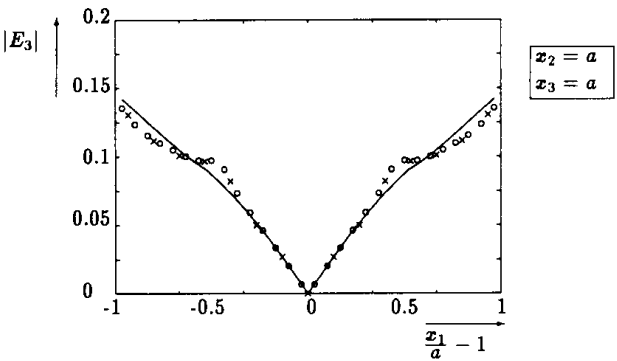

Fig. 3. The magnitude of the components of the total electric field inside a $15 \times 15 \times 15$ discretized lossy inhomogeneous sphere; $\epsilon_{r ; 1}=72, \sigma_{1}=$ $0.9 \mathrm{~S} / \mathrm{m}$ and $k_{0} a_{1}=0.163, \epsilon_{r ; 2}=7.5, \sigma_{2}=0.05 \mathrm{~S} / \mathrm{m}$ and $k_{0} a_{2}=0.314$. The numerical results pertaining to a mesh size of $15 \times 15 \times 15$ are presented by the symbols $\times$. The discretized object is refined with a mesh size of $30 \times 30 \times 30$ and the numerical results are presented by the symbols $\circ$. The analytical solution of the inhomogeneous sphere is presented by the solid line.

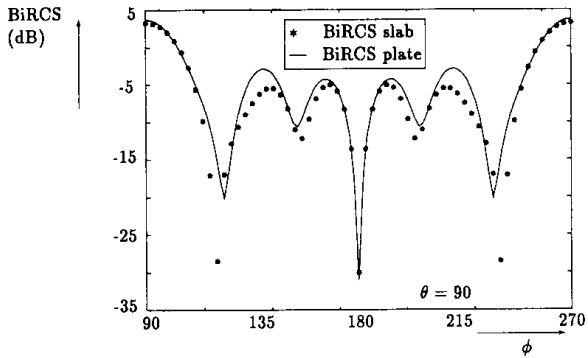

Fig 4. The bistatic radar cross section computed for the $2 \lambda_{0} \times 2 \lambda_{0}$ perfectly conducting plate is presented by the solid line and the bistatic radar cross section computed for the $2 \lambda_{0} \times 2 \lambda_{0} \times\left(2 \lambda_{0} / 31\right)$ slab with conductivity $1000 \mathrm{~S} / \mathrm{m}$ is presented by the symbols $*$. The frequency of operation is $100 \mathrm{MHz}$.

plane wave with the electric field vector parallel to the $x_{1}$-axis and propagating along the positive $x_{3}$-axis, hence in (49) we have

$$
\epsilon_{1}=1 \mathrm{~V} / \mathrm{m}, \quad \epsilon_{2}=0, \quad \epsilon_{3}=0,
$$




$$
\theta_{1}=0, \quad \theta_{2}=0, \quad \theta_{3}=+1 \text {. }
$$

The cube is subdivided with $M_{D^{s}}=N_{D^{s}}=P_{D^{s}}=7$. The number of iterations to obtain an error less than 0.1 percent is 38. In Fig. 5 we present the far-field for the dielectric cube. The solid curve is obtained using the present method, while the symbol ${ }^{\circ}$ represents the "trianglepatch" surface formulation developed by Rao (cf. [28]) and the symbol $\times$ represents the "pulse expansion point matching" volume formulation given by Sarkar et al. [28]. The far-fields are presented on a logarithmic vertical scale and all curves are normalized for $\theta=0$. From Fig. 5 it is observed that the far-fields that are computed using the present method agrees with the far-fields that are computed using the "triangle-patch" surface formulation, while the "pulse expansion point matching" volume formulation shows some discrepancies. As indicated by Sarkar et al. [28], the "pulse expansion point matching", volume formulation gives rise to spurious charge distributions influencing the accuracy of the near-field. As a consequence, the far-field shows some discrepancies. It is demonstrated that the weak formulation of the domainintegral equation does not suffer from this inaccuracy.

As fourth test case we consider a lossy dielectric cube to be present with its origin at $\boldsymbol{x}=\{a, a, a\}$, where the side length of the cube is equal to $2 a=0.75 \lambda_{0}\left(k_{0} a=\right.$ 2.3562 ). The conductivity is taken to be $1000 \mathrm{~S} / \mathrm{m}$ and the frequency of operation is taken to be $100 \mathrm{MHz}$. The cube is subdivided with $M_{D^{s}}=N_{D^{s}}=P_{D^{s}}=7$ and $M_{D^{s}}$ $=N_{D^{s}}=P_{D^{s}}=15$, respectively. The number of iterations to obtain an error less than 0.1 percent amounts to 68 and 189 for the $7 \times 7 \times 7$ subdivision and the $15 \times$ $15 \times 15$ subdivision, respectively. In Fig. 6 we present the bistatic radar cross section for the lossy dielectric cube. The dashed line represents the bistatic RCS results obtained for a subdivision of $7 \times 7 \times 7$, the solid line represents the bistatic RCS results obtained for a subdivision of $15 \times 15 \times 15$. The symbols $\circ$ represent the measured data given by Penno et al. [22] and the symbols * represent the CGM-FFT results of Catedra et al. [23]. It has been observed that the BiRCS obtained using the present method and the measured BiRCS results given by Penno et al. [22] agree very well, while Fig. 6 demonstrates that the weak formulation of the conjugate gradient FFT method produces more accurate results than the CGM-FFT implementation of Catedra et al. [23].

Finally, as fifth test case we consider a lossless dielectric cube to be present with its origin at $x=\{a, a, a\}$, where the side length of the cube is equal to $2 a=0.25 \lambda_{0}$ $\left(k_{0} a=0.7854\right)$. The relative permittivity is taken to be $\epsilon_{r}=4$. The frequency of operation is taken to be 100 $\mathrm{MHz}$. The cube is subdivided with $M_{D^{s}}=N_{D^{s}}=P_{D^{s}}=$ 15. The number of iterations to obtain an error less than 0.1 percent is 19. In Fig. 7 we present the bistatic radar cross section in the $\mathbf{E}$-plane $(\phi=0)$ and the bistatic radar cross section in the $\mathbf{H}$-plane $(\phi=90)$. The solid and dashed curves are obtained using the present method, while the symbols $*$ and + represent the results presented by Moheb and Shafai [29] for the E-plane and H-plane,
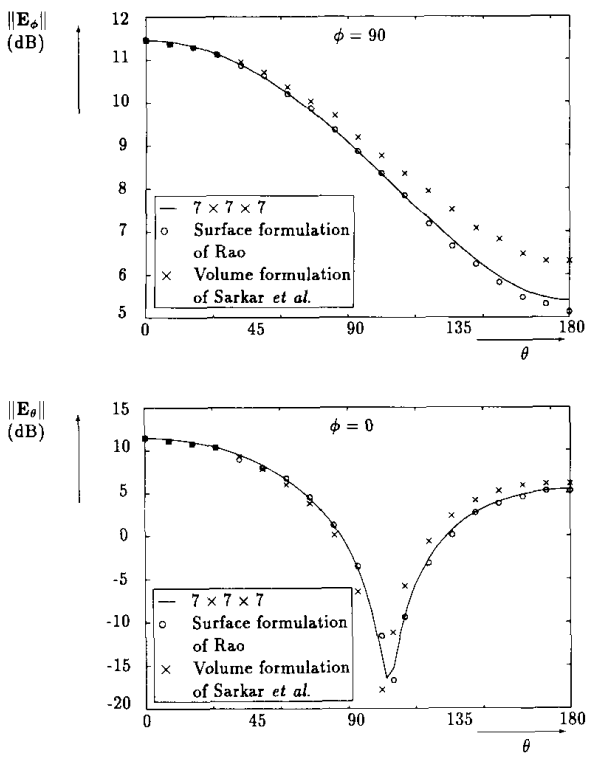

Fig. 5. The far fields as a function of $\theta$ computed for a lossless dielectric cube with $\epsilon_{r}=9, \sigma=0 \mathrm{~S} / \mathrm{m}$ and $k_{0} a=0.628319$. The solid lines represent the results obtained using the present method, the symbols $\circ$ represent the results obtained using the surface formulation and the symbols $x$ represent the results obtained using the volume formulation of Sarkar et al. [28].

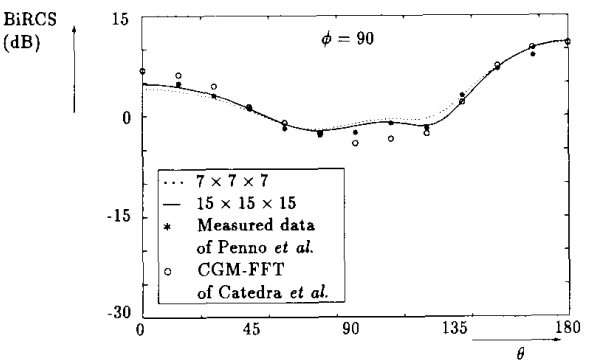

Fig. 6. The bistatic radar cross section as a function of $\phi$ computed for a lossy dielectric cube with $\epsilon_{r}=1 . \sigma=1000 \mathrm{~S} / \mathrm{m}$ and $k_{0} a=2.35610$. The results with a mesh size of $7 \times 7 \times 7$ are presented by the dashed line, while the results with a mesh size of $15 \times 15 \times 15$ are presented by the solid line. The symbols * represent the measured radar cross section given by Penno et al. [22] and the symbols represent the CGM-FFT results of Catedra et al. [23].

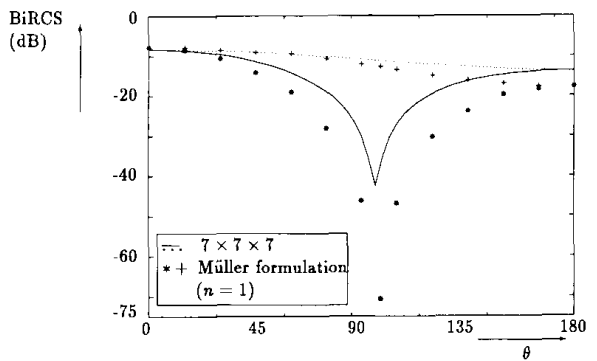

Fig. 7. The bistatic radar cross section as a function of $\theta$ computed for a lossless dielectric cube with $\epsilon_{r}=4, \sigma=0 \mathrm{~S} / \mathrm{m}$ and $k_{0} a=0.7854$. The results with a mesh size of $15 \times 15 \times 15$ are presented by the solid line and the approximated results given by Moheb and Shafai [29] obtained using the $n=1$ Müller formulation is presented by the symbols*. 
respectively. Moheb and Shafai have obtained their results using the first order Müller formulation $(n=1)$ and state that this approximation for the bistatic radar cross section is satisfactory. As observed from Fig. 7 their approximation of the bistatic radar cross section shows some (large) discrepancies.

\section{CONCLUSIONS}

In this paper we have presented a three-dimensional weak formulation of the conjugate gradient FFT method for dielectric scatterers. It is observed that the present weak formulation yields excellent agreement with the analytical results for the radially layered lossy dielectric sphere. Modeling the curved boundaries using a cubical mesh seems to be feasible and the discretization errors tend to vanish for increasingly finer discretizations. Comparison of the numerical results obtained using the weak formulation of the conjugate gradient FFT method with the numerical results obtained using other methods demonstrates that the present scheme produces accurate results.

Since we have maintained the simple scalar convolution structure of the vector potential, the computation time of our present method is even less than the computation time of the conjugate gradient FFT methods discussed in the Introduction. Further, it is noted that in contrast with the weak formulation of the two-dimensional TE scattering problems presented in [20], the present formulation allows the use of different mesh sizes in each Cartesian coordinate. The latter enhances the applicability of the weak formulation to complex, strongly inhomogeneous structures. Finally, it is mentioned that the extension of the present formulation to anisotropic objects is rather straightforward (see [25]).

\section{REFERENCES}

[1] J. H. Richmond, "Scattering by a dielectric cylinder of arbitrary cross section," IEEE Trans. Antennas Propagat., vol. AP-13, pp. 334341, Mar. 1965.

[2] — , "TE wave scattering by a dielectric cylinder of arbitrary crosssection shape," IEEE Trans. Antennas Propagat., vol. AP-14, pp. 460-464, July 1966.

[3] M. R. Hestenes and E. Stiefel, "Methods of conjugate gradient for solving linear systems, " $J$. Research of the National Bureau of Standard., vol. 49, pp. 409-435, 1952.

[4] P. M. van den Berg, "Iterative computational techniques in scattering based upon the integrated square error criterion," IEEE Trans. Antennas Propagat., vol. AP-32, pp. 1063-1071, Oct. 1984.

[5] N. N. Bojarski, "K-space formulation of the scattering problem in the time domain," J. Acoust. Soc. Am., vol. 72, pp. 570-584, 1982.

[6] T. K. Sarkar, E. Arvas and S. M. Rao, "Application of the Fast Fourier Transform and the conjugate method for efficient solution of electromagnetic scattering from both electrically large and small conducting bodies," Electromagnetics, vol. 5, pp. 99-122, 1985.

[7] - "Application of FFT and the conjugate method for the solution of electromagnetic radiation from electrically large and small conducting bodies," IEEE Trans. Antennas Propagat,, vol. AP-34, pp. 635-640, May 1986.

[8] R. Kastner and R. Mittra, "Iterative analysis of finite-sized planar frequency selective surfaces with rectangular patches or perforations," IEEE Trans. Antennas Propagat, vol. AP-35, pp. 372-377. Apr. 1987.

[9] R. Kastner, "A conjugate gradient procedure for the analysis of planar conductors with alternating patch and aperture formulation," IEEE Trans. Antennas Propagat, vol. 36, pp. 1616-1620. Nov. 1988.
[10] T. J. Peters and J. L. Volakis, "Application of a conjugate gradient FFT method to scattering from thin planar material plates," IEEE Trans. Antennas Propagat, vol. 36, pp. 518-526, Apr. 1988.

[11] T. A. Cwik and R. Mittra, "Scattering from a periodic array of freestanding arbitrarily shaped perfectly conducting or resistive patches,' IEEE Trans. Antennas Propagat., vol. AP-35, pp. 1226-1234, Nov. 1987.

[12] P. M. van den Berg, A. P. M. Zwamborn, G. C. Hsiao and R. E. Kleinman, "Iterative solutions of first order integral equations, " $\mathrm{Pe}$ ter Lang, Verlag, Berlin, 1991.

[13] C. Y. Shen, K. J. Glover, M. I. Sancer, and A. D. Varvatsis, "The discrete Fourier transform method of solving differential-integral equations in scattering theory," IEEE Trans. Antennas Propagat. vol. 37, pp. 1032-1041, August 1989

[14] A. P. M. Zwamborn and P. M. van den Berg, "The weak form of the conjugate gradient method for plate problems," IEEE Trans. Antennas Propagat., vol. 39, pp. 224-228, Feb. 1991.

[15] L. W. Pearson, "A technique for organizing Large moment calculations for use with iterative solution methods," IEEE Trans. Antennas Propagat., vol. AP-33, pp. 1031-1033, Sept. 1985.

[16] M. F. Catedra, J. G. Cuevas and L. Nuno, "A scheme to analyze conducting plates of resonant size using the conjugate gradient method and fast Fourier transform," IEEE Trans. Antennas Propagat., vol. 36, pp. 1744-1752, Dec. 1988.

[17] H. Massoudi, C. H. Durney and M. F. Iskander, "Limitations of the cubical block model of man in calculating SAR distributions," IEEE Trans. Microwave Theory Tech., vol. MTT-32, pp. 746-752, Aug. 1984

[18] M. J. Hagmann, "Comments on 'Limitations of the cubical block model of man in calculating SAR distributions," IEEE Trans. Microwave Theory Tech., vol. MTT-33, pp. 347-350, Apr. 1985.

[19] D. T. Borup, D. M. Sullivan and OM P. Gandi, "Comparison of the FFT conjugate gradient method and the finite-difference time-domain method for the 2-d absorption problem," IEEE Trans. Microwave Theory Tech., vol. MTT-35, pp. 383-395, Apr. 1987.

[20] A. P. M. Zwamborn, and P. M. van den Berg, "The weak form of the conjugate gradient method for 2-D TE scattering problems." IEEE Trans. Microwave Theory Tech., vol. 39, pp. 953-960, June 1991.

[21] N. Joachimowicz and C. Pichot, "Comparison of three formulations for the 2-D TE scattering problem." IEEE Trans. Microwave Theory Tech., vol. 38, pp. 178-185, Feb. 1990.

[22] R. Penno, P. K. Murthy, and G. A. Thiele, "Results of the application of the hybrid iterative method to scattering from a cube," IEEE Antennas Propagat. Soc. Int. Syp. Dig., Blacksburg, VA 1987. pp. 526-529.

[23] M. F. Catedra, E. Gago, and L. Nuno, "A numerical scheme to obtain the RCS of three-dimensional bodies of resonant size using the conjugate gradient method and the fast Fourier trnasform, "IEEE Trans. Antennas Propagat, vol. 37, pp. 528-537. May 1989.

[24] K. Barkeshli and J. L. Volakis, "On the implementation of the con jugate gradient Fourier transform method for scattering by planar plates," IEEE Trans. Antennas Propagat. Magazine, vol. 32, pp. 19-29, Apr. 1990

[25] A. P. M. Zwamborn, "'Scattering by objects with electric contrast,' Ph.D.-dissertation, Delft University Press, ISBN 90-6275-691-3/CIP, June 1991.

[26] C. A. Balanis, Advanced Engineering Electromagnetics. New York: Wiley, 1989.

[27] J. A. Kong, Electromagnetic Wave Theory. New York: Wiley, 1986

[28] T. K. Sarkar, E. Arvas and S. Ponnapalli, "Electromagnetic scatter ing from dielectric bodies," IEEE Trans. Antennas Propagat., vol. 37, pp. 673-676, May 1989.

[29] H. Moheb and L. Shafai, "Numerical solution of integral equations for dielectric objects of prismatic shapes," IEEE Trans. Antennas Propagat., vol. 39, pp. 758-766, June 1991.

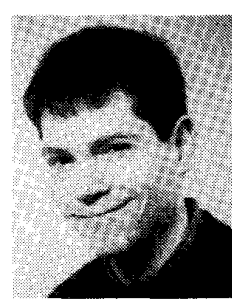

Peter Zwamborn was born in Schiedam, The Netherlands, on October 13, 1963. He received the M.Sc. degree in electrical engineering and the Ph.D. degree (Cum Laude) in technical sciences all from the Delft University of Technology in 1987 and 1991 , respectively. 


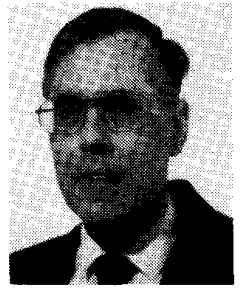

Peter M. van den Berg was born in Rotterdam, The Netherlands, on November 11, 1943. He received the degree in electrical engineering from the Polytechnical School of Rotterdam in 1964 the B.Sc. and M.Sc. degree in electrical engineering, and the Ph.D. degree in technical sci ences, all from the Delft University of Technology, in 1966, 1968, and 1971 , respectively.

From 1967 to 1968 , he was employed as a Research Engineer by the Dutch Patent Office. Since 1968 , he has been a member of the Scientific Staff of the Electromagnetic Research Group of the Delft University of Technology. During these years, he carried out research and taught classes in the area of wave propagation and scattering problems. During the academic year 1973-1974 he was Visiting Lecturer in the Department of Mathematics, University of Dundee, Scotland. During a three-month period in 1980 1981, he was Visiting Scientist at the Institute of Theoretical Physics, Goteborg, Sweden. He was appointed Professor at the Delft University of Technology in 1981. At present, his main research interest is the efficient computation of field problems using iterative techniques based on error minimization and the computation of fields in strongly inhomogeneous media. 